Pacific Journal of Mathematics

NORMS OF COMPACT PERTURBATIONS OF OPERATORS 


\section{NORMS OF COMPACT PERTURBATIONS OF OPERATORS}

\section{Catherine L. Olsen}

Let $\mathscr{B}(\mathscr{H})$ denote the algebra of all bounded linear operators on a complex separable Hilbert space. This paper is concerned with reducing the norm of a product of operators by compact perturbations of one or more of the factors. For any $T$ in $\mathscr{B}(\mathscr{H})$, it is well known that the infimum,

$$
\|T\|_{e}=\inf \{\|T+K\|: K \text { is a compact operator }\}
$$

is attained by some compact perturbation $T+K_{0}$. For $T$ a noncompact product of $n$ operators, $T=T_{1} \cdots T_{n}$, it is proved that this infimum can be obtained by a compact perturbation of any one of the factors. If $T$ is a compact product, so that the infimum is zero, it is shown that there are compact perturbations $T_{1}+K_{1}, \cdots, T_{n}+K_{n}$ of the factors of $T$ such that the product $\left(T_{1}+K_{1}\right) \cdots\left(T_{n}+K_{n}\right)$ is zero; furthermore, it may be necessary to perturb every factor of $T$ in order to obtain this zero infimum. These results are applied to an arbitrary operator $T$ to find a compact perturbation $T+K$ with $\left\|(T+K)^{2}\right\|=\left\|T^{2}\right\|_{e}$ and $\left\|(T+K)^{3}\right\|=\left\|T^{3}\right\|_{e}$; here the identical factors are perturbed in identical fashion to achieve both infima. Stronger theorems of this latter sort are proved for special classes of operators.

For any $T$ in $\mathscr{B}(\mathscr{H})$, let $\|T\|_{e}$ as defined above, be called the essential norm of $T$ [7]. I. C. Gohberg and M. G. Krein first showed in [4] that for any $T$ in $\mathscr{B}(\mathscr{H})$ there is a compact perturbation $T+K_{0}$ which realizes the essential norm (so $\left\|T+K_{0}\right\|=\|T\|_{e}$ ). The case $n=2$ of the theorem stated above for compact products was proved in a different way in [6]: for any compact product $T=T_{1} T_{2}$ of two factors, a projection $E$ was constructed so that $T_{1} E$ and $(I-E) T_{2}$ are both compact (and so that the product of perturbations $T_{1}(I-E)$ and $E T_{2}$ is zero).

This study was motivated partly by questions considered by $\mathbf{J}$. K. Plastiras and the author in [7]: if $T$ is a bounded operator on $\mathscr{H}$, is there a compact $K$ with $\|p(T+K)\|=\|p(T)\|_{e}$ for all complex polynomials $p$ ? Less ambitiously, if $T$ and $p$ are both given, is there a compact $K_{p}$ such that $\left\|p\left(T+K_{p}\right)\right\|=\|p(T)\|_{e}$ ? We know of no examples where either of these questions has a negative answer.

It follows from the results proved here on perturbations of products that for each $T$ in $\mathscr{B}(\mathscr{H})$, there is a compact $K$ with $\|T+K\|=\|T\|_{e}$ and $\left\|(T+K)^{2}\right\|=\left\|T^{2}\right\|_{e}$; and a compact $L$ with $\left\|(T+L)^{2}\right\|=\left\|T^{2}\right\|_{e}$ and $\left\|(T+L)^{3}\right\|=\left\|T^{3}\right\|_{e}$. If $T^{3}$ is not compact we can take $K=L$, to get one 
perturbation achieving all three essential norms. There appear to be erious difficulties in passing from $T^{3}$ to $T^{4}$. The existence of an operator $K$ as above was proved in [7] for any partial isometry $T$, and for certain other operators.

Stronger results are obtainable for special classes of operators. In [7] it was shown that for operators $T$ which are subnormal or essentially normal, there is one compact $K$ such that $\|p(T+K)\|=\|p(T)\|_{e}$, for every complex polynomial $p$. Here we prove this for $n$-normal operators. Turning to operators with no normality properties, we show that for any weighted shift $T$, there is one compact $K$ with $\left\|(T+K)^{n}\right\|=$ $\left\|T^{n}\right\|_{e}$ for all $n$. If in addition $T$ is nilpotent, then $\|p(T+K)\|=\|p(T)\|_{e}$ for every polynomial $p$. In [6] it was shown that for any $T$ in $\mathscr{B}(\mathscr{H})$ with $p(T)$ compact, there is a compact $K_{p}$ with $\left\|p\left(T+K_{p}\right)\right\|=\|p(T)\|_{e}=0$.

If it were true that every $T$ in $\mathscr{B}(\mathscr{H})$ could be perturbed by $K$, to simultaneously obtain $\|p(T+K)\|=\|p(T)\|_{e}$ for every polynomial $p$, this would have significant consequences. It would immediately imply the theorem of $T$. T. West [11] that every Riesz operator is a compact perturbation of a quasinilpotent, and would also answer a question of $\mathrm{W}$. Arveson: if $\pi(T)$ is quasialgebraic in the Calkin algebra, so that $\left\|p_{n}(\pi(T))\right\|^{1 / \operatorname{deg} p_{n}} \rightarrow 0$, then is there a compact $K$ so that $\| p_{n}(T+$ $K) \|^{1 / \operatorname{deg} p_{n}} \rightarrow 0$, for the same sequence $\left\{p_{n}\right\}_{n}$ of monic polynomials? A partial answer to this latter question, and further discussion is given in [7]. See also the question raised by S. R. Caradus [3].

In a recent communication we have learned that D. Legg, P. Smith, and $\mathrm{J}$. Ward have proved using Banach space techniques, that for any $T$ in $\mathscr{B}(\mathscr{H})$, there is one compact $K$ with $\|T+K+\lambda I\|=\|T+\lambda I\|_{\text {e, }}$ for all complex $\lambda$. Thus it is possible to simultaneously attain the essential norm for all linear polynomials in $T$.

A related result in a more general setting has been obtained by G. K. Pedersen [8]. In [7] it was shown for any $T$ in $\mathscr{B}(\mathscr{H})$ and for any polynomial $p$, that

$$
\|p(T)\|_{e}=\inf \|p(T+K)\|, K \text { compact. }
$$

Pedersen has proved that if $\mathscr{A}$ is a $C^{*}$-algebra and $\mathscr{I}$ is a closed ideal in $\mathscr{A}$ then for any $A \in \mathscr{A}$ and for any $n$,

$$
\left\|A^{n}+\mathscr{I}\right\|=\inf \left\|(A+B)^{n}\right\|, \quad B \in \mathscr{I} .
$$

Let $\mathscr{K}$ denote the closed two-sided ideal in $\mathscr{B}(\mathscr{H})$ of compact operators, and let $\pi: \mathscr{B}(\mathscr{H}) \rightarrow \mathscr{B}(\mathscr{H}) / \mathscr{K}$ be the natural homomorphism onto the quotient $C^{*}$-algebra, the Calkin algebra. Then the essential norm of $T$ in $\mathscr{B}(\mathscr{H})$ defined above is actually the $C^{*}$-norm of $\pi(T)$ in the Calkin algebra. We say that $D \in \mathscr{B}(\mathscr{H})$ is diagonal if there is an 
orthonormal basis for $\mathscr{H}$ consisting of eigenvectors for $D$. A finite rank operator is one with finite-dimensional range. The range projection of $T \in \mathscr{B}(\mathscr{H})$ is the smallest projection $Q$ such that $Q T=T$, and the support projection $P$ is the smallest projection such that $T P=$ $T$. Throughout the paper we use $|T|$ to denote $\left(T^{*} T\right)^{1 / 2}$ and $\sigma(T)$ to denote the spectrum of $T \in \mathscr{B}(\mathscr{H})$. The reader is referred to [5] for general facts about Hilbert space operators.

\section{Reducing the norm of a product by perturbing its}

factors. This first theorem is the heart of the paper.

THEOREM 1. Let $A, B$ in $\mathscr{B}(\mathscr{H})$ be such that the product $A B$ is not compact. Then there is a compact operator $K$ such that

$$
\|A(I-K) B\|=\|A B\|_{e}
$$

Furthermore, if $\left\{e_{n}\right\}_{n}$ is any orthonormal basis for $\mathscr{H}$, then $K$ can be constructed to be diagonal relative to that basis, with $0 \leqq K \leqq I$.

Before beginning the proof we make some relevant observations. If $D$ is any diagonal operator with $\|D\| \leqq 1$, then it is trivial that $\|D A B x\| \leqq\|A B x\|$, for any $x \in \mathscr{H}$ and any $A, B$ in $\mathscr{B}(\mathscr{H})$. It is also obvious that $\|A B D\| \leqq\|A B\|$, although $\|A B D x\| \leqq\|A B x\|$ may not hold for every $x$. On the other hand, there is no general relationship between $\|A D B\|$ and $\|A B\|$.

We remark also that this theorem is false if the product $A B$ is compact. To see this let $A$ be any injective compact operator and let $B=I$. Then $\|A B\|_{e}=0$, but $A(I-K) B$ cannot be zero if $K$ is any compact operator.

Proof. We may assume that $\|A\| \leqq 1$ and $\|B\| \leqq 1$. Let $\left\{P_{k}\right\}_{k}$ be the increasing sequence of finite rank projections with range $\left(P_{k}\right)=$ $\operatorname{span}\left\{e_{1}, \cdots, e_{k}\right\}$.

Let $\mu$ be any number with $\|A B\|_{e}<\mu<\|A B\|=\mu_{0}$. We will first construct a finite rank perturbation $D$ of $I$ so that $0 \leqq D \leqq I ; D$ will be $\left\{e_{n}\right\}_{n}$-diagonal; and with $\|A D B\| \leqq \mu$. Then we will show how this construction is repeated, to define by induction the desired operator $I-K$. In order to be able later to set up the induction, we will write in the factor $I$, which is being perturbed.

Let $E(\lambda)$ be the spectral resolution for $|A I B|$. Set $E=$ $E\left(\left(\mu-2 \delta, \mu_{0}\right]\right)$, where $\delta>0$ is a small number with $\mu-2 \delta>$ $\|A B\|_{e}$. Then $E$ must be a finite rank projection: otherwise, we could find an infinite orthonormal set $\left\{x_{n}\right\}_{n}$ such that $x_{n} \in \operatorname{ran}(E)$, and hence for which 


$$
\left\|A I B x_{n}\right\|=\left\||A I B| x_{n}\right\|>\mu-2 \delta .
$$

But this would imply

$$
\|A I B\|_{e} \geqq \mu-2 \delta>\|A I B\|_{e}
$$

a contradiction.

Let $G$ be the projection onto $\operatorname{ran}(I B E)$, so $G$ is finite rank. Choose $P_{k_{1}}$ from the sequence $\left\{P_{k}\right\}$ large enough so that

$$
\left\|\left(I-P_{k_{1}}\right) G\right\|<\nu
$$

where $\nu>0$ is a very small number to be determined. Let $Q_{1}$ be the finite rank projection onto $\operatorname{ran}\left(A P_{k_{1}}\right)$. Let $H_{1}$ be the support projection of the finite rank operator $Q_{1} A\left(I-P_{k_{1}}\right)$, so $H_{1} \leqq I-P_{k_{1}}$.

Choose $k_{2}>k_{1}$ sufficiently large so that

$$
\left\|Q_{1} A\left(I-P_{k_{2}}\right)\right\|=\left\|Q_{1} A\left(I-P_{k_{1}}\right) H_{1}\left(I-P_{k_{2}}\right)\right\| \leqq\left\|H_{1}\left(I-P_{k_{2}}\right)\right\|<\nu .
$$

Let $Q_{2}$ be the finite rank projection onto $\operatorname{ran}\left(A P_{k_{2}}\right)$. Let $H_{2}$ be the finite rank support projection of $Q_{2} A\left(I-P_{k_{2}}\right)$, so $H_{2} \leqq I-P_{k_{2}}$.

Choose $k_{3}>k_{2}$ sufficiently large so that

$$
\left\|Q_{2} A\left(I-P_{k_{3}}\right)\right\|=\left\|Q_{2} A\left(I-P_{k_{2}}\right) H_{2}\left(I-P_{k_{3}}\right)\right\| \leqq\left\|H_{2}\left(I-P_{k_{3}}\right)\right\|<\nu .
$$

Repeat this process $m$ times, where $m$ is to be determined, to get two increasing sets of finite rank projections $\left\{Q_{n}\right\}_{n=1}^{m},\left\{P_{k_{n}}\right\}_{n=1}^{m}$. Set $E_{1}=P_{k_{1}}$, $E_{2}=P_{k_{2}}-P_{k_{1}}, \cdots, E_{m}=P_{k_{m}}-P_{k_{m-1}}, \quad E_{m+1}=I-P_{k_{m}}$. Set $F_{1}=Q_{1}, \quad F_{2}=$ $Q_{2}-Q_{1}, \cdots, F_{m}=Q_{m}-Q_{m-1}, F_{m+1}=I-Q_{m}$.

Observe now that $F_{j} A E_{n}=0$, if $n<j$ : for,

$$
F_{j} A E_{n}=F_{j} A P_{k_{n}} E_{n}=F_{j} Q_{n} A P_{k_{n}} E_{n}=\left(Q_{j}-Q_{j-1}\right) Q_{n} A P_{k_{n}} E_{n}=0,
$$

whenever $n<j$.

Observe also that $\left\|F_{j} A E_{n}\right\|<\nu$ if $n>j+1$ for then $E_{n}=$ $\left(I-P_{k_{j+1}}\right) E_{n}$, so that

$$
\left\|F_{j} A E_{n}\right\|=\left\|F_{j} Q_{j} A\left(I-P_{k_{l+1}}\right) E_{n}\right\| \leqq\left\|Q_{j} A\left(I-P_{k_{j+1}}\right)\right\|<\nu .
$$

Now, set $\gamma=(\mu-2 \delta) /\|A I B\|$, so $0<\gamma<1$.

Define $D=I \sum_{j=1}^{m+1} \eta_{j} E_{j}$, where $\gamma=\eta_{1}<\eta_{2}<\cdots<\eta_{m+1}=1$, is an even partition of the interval $[\gamma, 1]$. We choose a small $\epsilon>0$ to be determined, and we now determine $m$ : so that $m \epsilon>1-\gamma$. In other words, $\eta_{j}-\eta_{j-1}<\epsilon$. Thus $D$ is a finite rank perturbation of $I$, and is $\left\{e_{n}\right\}_{n}$-diagonal. 
We will now show that $\|A D B\| \leqq \mu$. (Note that so far we have that $\gamma=\gamma(\delta, \mu)$, and $m=m(\gamma, \epsilon)$; but we are free to choose $\epsilon$ and $\nu$ as small as we wish.)

Let $z$ be a unit vector of $\mathscr{H}$, and write $z=\alpha x \oplus \beta y$, where $|\alpha|^{2}+|\beta|^{2}=1, \quad\|x\|=1=\|y\|, \quad$ and $\quad x \in \operatorname{ran} E\left(\left(\mu-2 \delta, \mu_{0}\right]\right), \quad y \in$ $\operatorname{ran} E([0, \mu-2 \delta])$. Since these are orthogonal spectral projections for $|A I B|$, this means $A I B x$ is orthogonal to $A I B y$. Now,

$$
\|A D B z\|^{2} \leqq|\alpha|^{2}\|A D B x\|^{2}+|\beta|^{2}\|A D B y\|^{2}+2|\alpha \beta \|\langle A D B x, A D B y\rangle|,
$$

and we consider the three summands separately.

First,

$$
\begin{aligned}
\|A D B x\| & =\left\|A \sum_{j=1}^{m+1} \eta_{j} E_{j} I B x\right\| \\
& \leqq\left\|A \sum_{j=1}^{m+1} \eta_{j} E_{j} G I B x\right\| \\
& \leqq\left\|A \eta_{1} E_{1} G I B x\right\|+\nu \quad\left(\left\|\left(I-E_{1}\right) G\right\|<\nu\right) \\
& \leqq \eta_{1}\|A G I B x\|+2 \nu \\
& =\gamma\|A I B x\|+2 \nu \quad\left(\eta_{1}=\gamma\right) \\
& \leqq \frac{\mu-2 \delta}{\|A I B\|}\|A I B x\|+2 \nu \\
& \leqq \mu-2 \delta+2 \nu .
\end{aligned}
$$

Now consider

$$
\begin{aligned}
\|A D B y\|= & \left\|A \sum_{n=1}^{m+1} \eta_{n} E_{n} I B y\right\|=\left\|\sum_{j=1}^{m+1} F_{j} A \sum_{n=1}^{m+1} \eta_{n} E_{n} I B y\right\| \\
= & \| F_{1} A \eta_{1} E_{1} I B y+F_{1} A \eta_{2} E_{2} I B y+F_{1} A \sum_{n=3}^{m+1} \eta_{n} E_{n} I B y \\
& +F_{2} A \eta_{2} E_{2} I B y+F_{2} A \eta_{3} E_{3} I B y+F_{2} A \sum_{n=4}^{m+1} \eta_{n} E_{n} I B y \\
& \vdots \\
& +F_{m-1} A \eta_{m-1} E_{m-1} I B y+F_{m-1} A \eta_{m} E_{m} I B y \\
& +F_{m-1} A \eta_{m+1} E_{m+1} I B y \\
& +F_{m} A \eta_{m} E_{m} I B y+F_{m} A \eta_{m+1} E_{m+1} I B y \\
& +F_{m+1} A \eta_{m+1} E_{m+1} I B y \|
\end{aligned}
$$

since $F_{j} A E_{n}=0$ if $n<j$; 


$$
\begin{aligned}
\leqq & \| \sum_{j=1}^{m} F_{j} A \eta_{j}\left(E_{j}+E_{j+1}\right) I B y+\sum_{j=1}^{m}\left(\eta_{j+1}-\eta_{j}\right) F_{j} A E_{j+1} I B y \\
& +F_{m+1} A \eta_{m+1} E_{m+1} I B y \|+\frac{m(m-1)}{2} \nu,
\end{aligned}
$$

since $\left\|F_{j} A E_{n}\right\|<\nu$ if $n>j+1$;

$$
\begin{gathered}
\leqq\left\|\sum_{j=1}^{m+1} \eta_{j} F_{j} A I B y-\sum_{j=1}^{m-1} \eta_{j} F_{j} A \sum_{n=j+2}^{m+1} E_{n} I B y\right\| \\
+\epsilon\left\|\sum_{j=1}^{m} F_{j} A E_{j+1} I B y\right\| \\
+\frac{m(m-1)}{2} \nu,
\end{gathered}
$$

since $\eta_{j+1}-\eta_{j}<\epsilon$

$$
\begin{aligned}
& \leqq\left\|\sum_{j=1}^{m+1} \eta_{j} F_{j} A I B y\right\|+m(m-1) \nu+\epsilon \\
& \leqq\|A I B y\|+m(m-1) \nu+\epsilon \\
& \leqq \mu-2 \delta+m(m-1) \nu+\epsilon .
\end{aligned}
$$

Finally, consider

$$
\begin{aligned}
|\langle A D B x, A D B y\rangle| & =\left|\left\langle A \sum_{j=1}^{m+1} \eta_{j} E_{j} I B x, A D B y\right\rangle\right| \\
& \leqq\left|\left\langle A \eta_{1} E_{1} G I B x, A D B y\right\rangle\right| \\
& \quad+\left|\left\langle A \sum_{j=2}^{m+1} \eta_{j} E_{j}\left(I-P_{k_{1}}\right) G I B x, A D B y\right\rangle\right| \\
& \leqq \\
& \left|\left\langle F_{1} A \eta_{1} E_{1} G I B x, F_{1} A \sum_{n=1}^{m+1} \eta_{n} E_{n} I B y\right\rangle\right|+\nu,
\end{aligned}
$$

since $F_{1} A E_{1}=A E_{1}$, and $\left\|\left(1-P_{k_{1}}\right) G\right\|<\nu$;

$$
=\left|\left\langle F_{1} A \eta_{1} E_{1} G I B x, F_{1} A\left(\eta_{1} E_{1}+\eta_{2} E_{2}\right) I B y\right\rangle\right|+(m-1) \nu,
$$

since $\left\|F_{j} A E_{n}\right\|<\nu$ if $n \geqq j+2$;

$$
\begin{aligned}
& \leqq\left|\left\langle F_{1} A \eta_{1} E_{1} G I B x, F_{1} A \eta_{1}\left(E_{1}+E_{2}\right) I B y\right\rangle\right|+\epsilon+(m-1) \nu \\
& \leqq\left|\left\langle F_{1} A E_{1} G I B x, F_{1} A\left(E_{1}+E_{2}\right) I B y\right\rangle\right|+\epsilon+(m-1) \nu \\
& \leqq\left|\left\langle F_{1} A E_{1} G I B x, F_{1} A \sum_{n=1}^{m+1} E_{n} I B y\right\rangle\right|+\epsilon+(2 m-2) \nu \\
& =\left|\left\langle A E_{1} G I B x, A I B y\right\rangle\right|+\epsilon+(2 m-2) \nu \\
& \leqq|\langle A G I B x, A I B y\rangle|+\epsilon+2 m \nu \\
& =\epsilon+2 m \nu .
\end{aligned}
$$


Now determine $\epsilon=\epsilon(\delta, \mu)$ sufficiently small $\left(2 \epsilon<\delta\right.$ and $(\mu-\delta)^{2}+2 \epsilon<$ $\left.\mu^{2}\right)$, and $\nu=\nu(m, \epsilon)$ sufficiently small $\left(2 m \nu<\epsilon, \nu<\epsilon, m^{2} \nu<\epsilon\right)$, so that

$$
\begin{aligned}
\|A D B z\|^{2} & \leqq|\alpha|^{2}\|A D B x\|^{2}+|\beta|\|A D B y\|^{2}+2|\alpha \beta||\langle A D B x, A D B y\rangle| \\
& <|\alpha|^{2}(\mu-\delta)^{2}+|\beta|^{2}(\mu-\delta)^{2}+2|\alpha \beta| 2 \epsilon \\
& <(\mu-\delta)^{2}+2 \epsilon \\
& <\mu^{2}
\end{aligned}
$$

Thus we have $D$ with the desired properties.

This construction is the first step in an induction. To view it as such, rename $D=D_{1}, \mu=\mu_{1}, \delta=\delta_{1}, \gamma=\gamma_{1}, m=m_{1}, \epsilon=\epsilon_{1}, \nu=\nu_{1}$, $\left\{E_{j}\right\}_{i=1}^{m+1}$ as $\left\{E_{1 j}\right\}_{i=1}^{m_{1}+1}$, and $\left\{\eta_{i}\right\}_{i=1}^{m+1}$ as $\left\{\eta_{1 j}\right\}_{i=1}^{m_{1}+1}$. A decreasing sequence $\left\{D_{n}\right\}_{n}$ of $\left\{e_{n}\right\}_{n}$-diagonal operators will be constructed by induction; each a finite rank perturbation of $I$. Then the operator $D_{0}=\inf D_{n}$, will be the desired compact perturbation, $D_{0}=I-K$.

We specify the sequences of constants to be used (the first terms as above):

(1) Choose a strictly decreasing sequence of positive numbers $\left\{u_{n}\right\}_{n}$ with $\mu_{1}$ (as above) $<\mu_{0}=\|A B\|$ and $\lim \mu_{n}=\|A B\|_{e} \neq 0$. The sequence $\left\{D_{n}\right\}_{n}$ will satisfy $\left\|A D_{n} B\right\| \leqq \mu_{n}$.

(2) Choose $\left\{\delta_{n}\right\}_{n}$ positive numbers decreasing to zero, so that $2 \delta_{n}<\mu_{n}-\mu_{n+1}$.

(3) Let $\left\{\gamma_{n}\right\}_{n}$ be the positive sequence converging to 1 given by $\gamma_{n}=\left(\mu_{n}-2 \delta_{n}\right) / \mu_{n-1}$.

Then from (2) we have

$$
\frac{\mu_{n+1}}{\mu_{n-1}}<\gamma_{n}<\frac{\mu_{n}}{\mu_{n-1}}
$$

so that the infinite product $\Pi \gamma_{n}$ converges to a nonzero limit precisely when the operator $A B$ is not compact; i.e., when the $\lim \mu_{n}=\|A B\|_{e} \neq 0$.

(4) Choose $\left\{\epsilon_{n}\right\}_{n}$ decreasing to zero, such that $2 \epsilon_{n}<\delta_{n}$, and $\left(\mu_{n}-\delta_{n}\right)^{2}+2 \epsilon_{n}<\mu_{n}^{2}$.

(5) Choose integers $\left\{m_{n}\right\}_{n}$ such that $1-\gamma_{n}<m_{n} \epsilon_{n}$.

(6) Finally, choose positive $\left\{\nu_{n}\right\}_{n}$ converging to zero, so that $\nu_{n}<\epsilon_{n}$, $m_{n}^{2} \nu_{n}<\epsilon_{n}$, and $2 m_{n} \nu_{n}<\epsilon_{n}$.

Now repeat the above construction, line for line, with $D_{1}$ in place of $I$, using $\mu_{2}, \delta_{2}, \epsilon_{2}, m_{2}, \nu_{2}$, and specifying $\left\{E_{2 i}\right\}_{i=1}^{m_{2}+1}$ and $\left\{\eta_{2 i}\right\}_{i=1}^{m_{2}+1}$; the only additional stipulation being that we choose $E_{21}>\sum_{i=1}^{m_{1}} E_{1 i}$. Thus we obtain a $D_{2} \leqq D_{1}, D_{2}$ a finite rank perturbation of $D_{1}$, and hence of $I$, with $\left\|A D_{2} B\right\|<\mu_{2}$ : 


$$
\begin{aligned}
D_{2} & =D_{1} \sum_{j=1}^{m_{2}+1} \eta_{2 j} E_{2 j}=\left[\sum_{i=1}^{m_{1}+1} \eta_{1 i} E_{1 \imath}\right]\left[\sum_{j=1}^{m_{2}+1} \eta_{2 j} E_{2 \jmath}\right] \\
& =\left[\sum_{i=1}^{m_{1}} \eta_{1 i} E_{1 \imath}\right] \eta_{21} E_{21}+E_{1, m_{1}+1}\left[\sum_{j=1}^{m_{2}+1} \eta_{2 j} E_{2 j}\right] \\
& =\gamma_{2} \sum_{i=1}^{m_{1}} \eta_{1 \imath} E_{1 i}+\eta_{21}\left(E_{21}-\sum_{i=1}^{m_{1}} E_{1 i}\right)+\sum_{j=2}^{m_{2}+1} \eta_{2 j} E_{2 j}
\end{aligned}
$$

recalling that $\gamma_{2}$ is $\eta_{21}$. The point of this equation is to exhibit the diagonal operator $D_{2}$ as a linear combination of orthogonal projections.

Assume for induction we have recursively constructed $D_{1} \geqq D_{2} \geqq$ $\cdots \geqq D_{k-1}$ as above using in turn the specified constants and such that

$$
E_{j 1}>\sum_{i=1}^{m_{j-1}} E_{j-1, l}, \quad j=1, \cdots, k-1 .
$$

Then repeat the above construction with the $k$ th constants, choosing

$$
E_{k 1}>\sum_{i=1}^{m_{k-1}} E_{k-1, i}
$$

to obtain $\left\|A D_{k} B\right\| \leqq \mu_{k}$, and $D_{k} \leqq D_{k-1}$, where, as an orthogonal sum, we have

$$
\begin{aligned}
D_{k}= & \prod_{\jmath=2}^{k} \gamma_{\jmath}\left[\eta_{11}\left(E_{11}-0\right)+\sum_{i=2}^{m_{1}} \eta_{1 i} E_{1 i}\right] \\
& +\prod_{j=3}^{k} \gamma_{\jmath}\left[\eta_{21}\left(E_{21}-\sum_{i=1}^{m_{1}} E_{1 i}\right)+\sum_{i=2}^{m_{2}} \eta_{2 i} E_{2 i}\right] \\
& \vdots \\
& +1\left[\eta_{k 1}\left(E_{k 1}-\sum_{i=1}^{m_{k-1}} E_{k-1, i}\right)+\sum_{i=2}^{m_{k}} \eta_{k i} E_{k i}\right] \\
& +\left[I-\sum_{i=1}^{m_{k}} E_{k i}\right],
\end{aligned}
$$

noting that the last summand equals $\eta_{k, m_{k}+1} E_{k, m_{k}+1}$.

By induction we now have the desired sequence $\left\{D_{n}\right\}_{n}$ defined. We show that $\left\{D_{n}\right\}_{n}$ converges uniformly to inf $D_{n}=D_{0}$, with

$$
D_{0}=\sum_{n=1}^{\infty}\left(\prod_{j=n+1}^{\infty} \gamma_{j}\right)\left[\eta_{n 1}\left(E_{n 1}-\sum_{i=1}^{m_{n-1}} E_{n-1, i}\right)+\sum_{i=2}^{m_{n}} \eta_{n i} E_{n i}\right]
$$

(where $E_{0 t}=0$, all $i$ ). This will complete the proof: for then, $A D_{n} B$ converges to $A D_{0} B$, so that $\left\|A D_{n} B\right\| \leqq \mu_{n}$ each $n$, implying that 
$\left\|A D_{0} B\right\| \leqq \lim \mu_{n}=\mu$. And since $I-D_{n}$ is finite rank for each $n$, therefore $I-D_{0}$ must be compact. Then $K=I-D_{0}$ will satisfy the conclusion of the theorem.

The convergence of $\left\{D_{n}\right\}$ follows simply because the product $\Pi \gamma_{j}$ converges. That is,

$$
\begin{aligned}
D_{k}-D_{0}= & \left\{\left(1-\prod_{j=k+1}^{\infty} \gamma_{j}\right) \prod_{j=2}^{k} \gamma_{j}\left[\eta_{11} E_{11}+\sum_{i=2}^{m_{1}} \eta_{1 i} E_{1 i}\right]\right. \\
& +\left(1-\prod_{j=k+1}^{\infty} \gamma_{j}\right) \prod_{j=3}^{k} \gamma_{j}\left[\eta_{21}\left(E_{21}-\sum_{i=1}^{m_{1}} E_{1 i}\right)+\sum_{i=2}^{m_{2}} \eta_{2 i} E_{2 i}\right] \\
& \vdots \\
& \left.+\left(1-\prod_{j=k+1}^{\infty} \gamma_{j}\right)(1)\left[\eta_{k 1}\left(E_{k 1}-\sum_{i=1}^{m_{k-1}} E_{k-1, i}\right)+\sum_{i=2}^{m_{k}} \eta_{k i} E_{k i}\right]\right\} \\
& +\sum_{n=k+1}^{\infty}\left\{\left(1-\prod_{j=n}^{\infty} \gamma_{j}\right)\left(E_{n 1}-\sum_{i=1}^{m_{n-1}} E_{n-1, i}\right)\right. \\
& \left.+\sum_{i=2}^{m_{n}}\left[1-\left(\prod_{j=n+1}^{\infty} \gamma_{j}\right) \eta_{n i}\right] E_{n i}\right\}
\end{aligned}
$$

(recall $\left.\gamma_{n}=\eta_{n 1}\right)$. Note that for each $n$,

$$
1-\left(\prod_{j=n+1}^{\infty} \gamma_{j}\right) \eta_{n i}<1-\prod_{j=n}^{\infty} \gamma_{l}
$$

Thus,

$$
\left\|D_{k}-D_{0}\right\| \leqq \sup _{n \geqq k+1}\left(1-\prod_{j=n}^{\infty} \gamma_{j}\right)\|R\|
$$

where $R$ is a sum of orthogonal projections multiplied by constants that are between zero and one. Thus $\lim _{k}\left\|D_{k}-D_{0}\right\|=0$, and the theorem is proved.

As immediate corollaries, we get the following:

THEOREM 2. For any $A, B$ in $\mathscr{B}(\mathscr{H})$, and any $\epsilon>0$, there is a finite rank operator $F$ with $0 \leqq F \leqq I$ such that

$$
\|A(I-F) B\|<\|A B\|_{e}+\epsilon \text {. }
$$

Furthermore, given any orthonormal basis, F can be constructed to be diagonal relative to that basis.

Proof. This is simply the first construction in the preceding proof, and it does not require noncompactness of the product $A B$. 
Theorem 3. Let $T_{1}, \cdots, T_{n}$ be in $\mathscr{B}(\mathscr{H})$ such that $\Pi T_{j}$ is not compact. Then for any $j$ there is a compact perturbation $S_{j}$ of $T_{j}$ such that

$$
\left\|T_{1} \cdots T_{j-1} S_{j} T_{j+1} \cdots T_{n}\right\|=\left\|\Pi T_{j}\right\|_{e}
$$

If $T_{1}$ is diagonal, $S_{j}$ may be obtained by reducing the moduli of some eigenvalues of $T_{j}$.

Proof. For $j=1$, set $A=I, B=\Pi T_{j}$ and apply Theorem 1 to get a compact $K$ with $\left\|(I-K) \Pi T_{j}\right\|=\left\|\Pi T_{j}\right\|_{e}$. Then set $S_{1}=(I-K) T_{1}$. If $T_{1}$ is diagonal, construct $K$ to be diagonal relative to the same basis as $T_{1}$. If $j=2$, set $A=T_{1}$ and $B=\Pi_{j>1} T_{j}$, and proceed similarly; the other cases are the same.

In order to obtain a corresponding theorem for compact products of operators we require some preliminary results.

Proposition 4. Any $T$ in $\mathscr{B}(\mathscr{H})$ has a compact perturbation $S$ where $|S|$ is diagonal.

Proof. Let $T=U|T|$ be the polar decomposition for $T$. Let $E=U^{*} U$ and regard $|T|$ as a positive operator in $\mathscr{B}(E \mathscr{H})$. By a theorem of $\mathrm{H}$. Weyl [10], there is a compact operator $K$ in $\mathscr{B}(E \mathscr{H})$ with $|T|+K$ diagonal relative to some orthonormal basis for $E \mathscr{H}$. Consider this as a diagonal operator on $\mathscr{H}: \sigma(|T|+K)$ is the closure of the set $\left\{d_{n}\right\}_{n}$ of diagonal entries. The Weyl spectrum of $|T|+K$ is

$$
\sigma_{w}(|T|+K)=\bigcap_{\text {compact }} \sigma(|T|+K+C)
$$

Since $|T|+K$ is normal, by Weyl's Theorem, $\sigma_{w}(|T|+K)$ consists of the cluster points of $\sigma(|T|+K)$ union the eigenvalues that are repeated infinitely often [1]. Now,

$$
\sigma_{w}(|T|+K)=\sigma_{w}(|T|) \subset \sigma(|T|),
$$

so $\sigma_{w}(|T|+K)$ consists of nonnegative real numbers. Thus the subset of $\left\{d_{n}\right\}_{n}$ consisting of nonzero, nonpositive numbers has no accumulation points and no infinitely repeated numbers. If we replace such $d_{n}$ by the element of $\sigma_{w}(|T|+K)$ nearest $d_{n}$, the result is a positive diagonal operator $D$ which is a compact perturbation of $|T|+K$, and such that $E D=D$. Then $S=U D$ is the desired compact perturbation of $T$. 
Proposition 5. Let $A, B$ be in $\mathscr{B}(\mathscr{H})$ and let $K$ be any compact operator. There are compact perturbations $A^{\prime}$ and $B^{\prime}$ of $A$ and $B$ and $a$ projection $E$ such that

$$
A^{\prime} B^{\prime}=(A B+K) E
$$

Proof. Let $U|B|$ be the polar decomposition for $B$. Using the previous result, assume that $|B|$ is diagonal relative to an orthonormal basis $\left\{e_{n}\right\}_{n}$, with diagonal sequence $\left\{b_{n}\right\}_{n}$.

To motivate the proof, we remark that, since $B$ may not be invertible, we cannot simply set $A^{\prime}=A+K B^{-1}$, to get $A^{\prime} B=$ $A B+K$. However, if we first erase a subsequence of $\left\{b_{n}\right\}_{n}$ which converges to zero "too fast", then this approach will work.

Let $P_{n}$ be the finite rank projection onto span $\left\{e_{1}, \cdots, e_{n}\right\}$. Then $\left\{P_{n} K P_{n}\right\}_{n}$ converges uniformly to $K$, so choose a subsequence $\left\{P_{n_{k}}\right\}_{k}$ with

$$
\left\|K-P_{n_{k}} K P_{n_{k}}\right\|<\frac{1}{2^{2 k}}
$$

Define a sequence of nonnegative real numbers $\left\{c_{m}\right\}_{m}$ by

$$
c_{m}= \begin{cases}0 \quad \text { if } \quad b_{m}<\frac{1}{2^{k}} \\ b_{m} \quad \text { if } \quad b_{m} \geqq \frac{1}{2^{k}}\end{cases}
$$

whenever $n_{k-1}<m \leqq n_{k}$, for $k=1,2, \cdots$, and $n_{0}=0$. Define another sequence $\left\{d_{m}\right\}_{m}$ by

$$
d_{m}=\left\{\begin{array}{lll}
1 & \text { if } \quad c_{m}=0 \\
\frac{1}{c_{m}} & \text { if } \quad c_{m} \neq 0
\end{array}\right.
$$

Note that for $m \leqq n_{k}, d_{m} \leqq 2^{k}$. Let $C \in \mathscr{B}(\mathscr{H})$ be the diagonal operator with diagonal $\left\{c_{m}\right\}_{m}$ relative to $\left\{e_{m}\right\}_{m}$, and let $D$ be the unbounded densely defined diagonal operator with diagonal $\left\{d_{m}\right\}_{m}$ relative to $\left\{e_{m}\right\}_{m}$. Clearly $|B|-C$ is a compact operator.

Furthermore $K D$ is a compact operator: in particular, the sequence $\left\{P_{n_{k}} K D P_{n_{k}}\right\}$ is uniformly Cauchy. For, assuming $k>i$, 


$$
\begin{aligned}
\left\|P_{n_{k}} K D P_{n_{k}}-P_{n_{i}} K D P_{n_{i}}\right\| & \leqq \sum_{j=i+1}^{k}\left\|P_{n_{j}} K D P_{n_{j}}-P_{n_{j-1}} K D P_{n_{j-1}}\right\| \\
& \leqq \sum_{j=i+1}^{k}\left\|P_{n_{j}}\left(K-P_{n_{j-1}} K P_{n_{j-1}}\right) D P_{n_{j}}\right\| \\
& \leqq \sum_{j=i+1}^{k}\left\|K-P_{n_{j-1}} K P_{n_{j-1}}\right\|\left\|D P_{n_{j}}\right\| \\
& \leqq \sum_{j=i+1}^{k} \frac{1}{2^{2(j-1)}} 2^{j}=\sum_{j=i+1}^{k} \frac{1}{2^{j-2}}<\frac{1}{2^{i-2}} .
\end{aligned}
$$

Since $\left\{P_{n_{k}}\right\}$ converges strongly to $I$, then $\left\{P_{n k} K D P_{n_{k}}\right\}$ converges uniformly to $K D$.

Let $E$ be the projection whose range is $\overline{\operatorname{span}}\left\{e_{n}: c_{n} \neq 0\right\}$. Thus $C=|B| E$ and $D C=E$.

To finish the proof, set $A^{\prime}=A+K D U^{*}, B^{\prime}=U C$. Then

$$
\begin{aligned}
A^{\prime} B^{\prime}=\left(A+K D U^{*}\right)(U C)=A U C+K D C=A U|B| E+K E & =(A B+K) E
\end{aligned}
$$

and we are done.

Using Theorem 2 , it is possible to reduce the norm of a compact product by perturbing any one factor. However it may be necessary to perturb every factor to get a zero product. For example, let $C$ be any one-to-one compact operator, and let $A=\left(\begin{array}{cc}I & 0 \\ 0 & C\end{array}\right), B=\left(\begin{array}{cc}C & 0 \\ 0 & I\end{array}\right), A B=$ $\left(\begin{array}{ll}C & 0 \\ 0 & C\end{array}\right)$, and let $\left(\begin{array}{cc}K & L \\ M & N\end{array}\right)$ be any compact operator. Then

$$
\left[A+\left(\begin{array}{cc}
K & L \\
M & N
\end{array}\right)\right] B=\left(\begin{array}{cc}
C+K C & L \\
M C & N+C
\end{array}\right)
$$

which equals zero only if $C(I+K)=0$, an impossibility. Thus the next theorem is the best possible general result.

THEOREM 6. Let $T_{1}, \cdots, T_{n}$ be in $\mathscr{B}(\mathscr{H})$ such that $\Pi T_{j}$ is compact. Then there are compact perturbations $S_{1}, \cdots, S_{n}$ of $T_{1}, \cdots, T_{n}$ with $\Pi S_{j}=0$.

Proof. The product $T_{1}\left(\Pi_{j=2}^{n} T_{j}\right)=C$, a compact operator.

By the previous proposition, there are compact $K_{1}, L_{1}$ and a projection $E_{1}$ with

$$
\left(T_{1}+K_{1}\right)\left(\prod_{j=2}^{n} T_{j}+L_{1}\right)=\left[T_{1}\left(\prod_{j=2}^{n} T_{j}\right)-C\right] E_{1}=0
$$


Now apply the proposition to $T_{2}\left(\Pi_{j=3}^{n} T_{j}\right)+L_{1}$ to get compact $K_{2}$ and $L_{2}$ and a projection $E_{2}$ with

$$
\left(T_{2}+K_{2}\right)\left(\prod_{j=3}^{n} T_{j}+L_{2}\right)=\left[T_{2}\left(\prod_{j=3}^{n} T_{j}\right)+L_{1}\right] E_{2}
$$

Thus

$$
\left(T_{1}+K_{1}\right)\left(T_{2}+K_{2}\right)\left(\prod_{j=3}^{n} T_{j}+L_{2}\right)=\left(\prod_{j=1}^{n} T_{j}-C\right) E_{1} E_{2}=0 .
$$

Repeated applications of the proposition yield:

$$
\prod_{j=1}^{n-2}\left(T_{j}+K_{j}\right)\left(T_{n-1} T_{n}+L_{n-2}\right)=\left(\prod_{j=1}^{n} T_{j}-C\right) \prod_{i=1}^{n-2} E_{i}=0 .
$$

And, a final application gives compact $K_{n-1}$ and $L_{n-1}$, and a projection $E_{n-1}$ with

$$
\left(T_{n-1}+K_{n-1}\right)\left(T_{n}+L_{n-1}\right)=\left(T_{n-1} T_{n}+L_{n-2}\right) E_{n-1}
$$

so that for $K_{n}=L_{n-1}$, we have

$$
\prod_{j=1}^{n}\left(T_{j}+K_{j}\right)=\prod_{j=1}^{n-2}\left(T_{j}+K_{j}\right)\left(T_{n-1} T_{n}+L_{n-2}\right) E_{n-1}=0
$$

and the theorem is proved.

2. Attaining the essential norm for polynomials in an operator. In this section we first show that any bounded operator can be perturbed to attain $\|T\|_{e},\left\|T^{2}\right\|_{e}$, or $\left\|T^{3}\right\|_{e}$; in most cases all three norms are achieved by a single compact perturbation of $T$. We then consider special classes of operators, weighted shifts and $n$-normal operators, for which stronger results are obtained. The first theorem follows by repeated applications of Theorem 3 .

THEOREM 7. Any $T$ in $\mathscr{B}(\mathscr{H})$ with $T^{3}$ not compact has a compact perturbation $S$ with $\|S\|=\|T\|_{e},\left\|S^{2}\right\|=\left\|T^{2}\right\|_{e}$ and $\left\|S^{3}\right\|=\left\|T^{3}\right\|_{e}$.

Proof. Using Proposition 4, we may assume that $|T|$ is diagonal, where $U|T|$ is the polar decomposition for $T$. Assume also $\|T\| \leqq 1$.

Let $\left\{\lambda_{n}\right\}_{n}$ be the sequence of diagonal entries of $|T|$ such that $\lambda_{n}>\||T|\|_{e}=\|T\|_{e}$ : then, $\lim \lambda_{n}=\|T\|_{e}$. Obtain a compact perturbation $T_{1}$ of $T$ by replacing each $\lambda_{n}$ with $\|T\|_{e}$, to get $\left|T_{1}\right|$ from $|T|$, and then setting $T_{1}=U\left|T_{1}\right|$. Clearly $\left\|T_{1}\right\|=\|T\|_{e}$. 
Now apply Theorem 3 to the product $T_{1}^{2}=U\left|T_{1}\right| T_{1}$, to get a compact perturbation $\left|T_{1}\right|^{\prime}$ of $\left|T_{1}\right|$ by reducing some of the eigenvalues of $\left|T_{1}\right|$, such that

$$
\left\|U\left|T_{1}\right|^{\prime} T_{1}\right\|=\left\|U\left|T_{1}\right| T_{1}\right\|_{e}=\left\|T^{2}\right\|_{e} .
$$

Since reducing the eigenvalues in a diagonal first or last factor does not raise the norm of a product, we have

$$
\left\|U\left|T_{1}\right|^{\prime} U\left|T_{1}\right|^{\prime}\right\| \leqq\left\|U\left|T_{1}\right|^{\prime} U\left|T_{1}\right|\right\|=\left\|T^{2}\right\|_{e}
$$

So let $T_{2}=U\left|T_{1}\right|^{\prime}$ (then $\left|T_{2}\right|=\left|T_{1}\right|^{\prime}$ ).

Finally, apply Theorem 3 to the product $\left|T_{2}\right| U\left|T_{2}\right| T_{2}$ to get a compact perturbation $\left|T_{2}\right|^{\prime}$ of $\left|T_{2}\right|$ by reducing some of the eigenvalues of $\left|T_{2}\right|$, such that

$$
\left\|\left|T_{2}\right| U\left|T_{2}\right|^{\prime} T_{2}\right\|=\left\|\left|T_{2}\right| U\left|T_{2}\right| T_{2}\right\|_{e}=\left\|U\left|T_{2}\right| U\left|T_{2}\right| T_{2}\right\|_{e}=\left\|T^{3}\right\|_{e},
$$

since $U^{*} U\left|T_{2}\right|=\left|T_{2}\right|$. Thus

$$
\left\|\left(U\left|T_{2}\right|^{\prime}\right)^{3}\right\|=\left\|\left|T_{2}\right|^{\prime} U\left|T_{2}\right|^{\prime} U\left|T_{2}\right|^{\prime}\right\| \leqq\left\|\left|T_{2}\right| U\left|T_{2}\right|^{\prime} T_{2}\right\|=\left\|T^{3}\right\|_{e}
$$

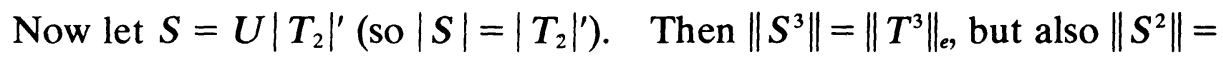
$\left\|T^{2}\right\|_{e}$, for,

$$
\left\|S^{2}\right\|=\left\|\left|T_{2}\right|^{\prime} U\left|T_{2}\right|^{\prime}\right\| \leqq\left\|\left|T_{2}\right| U\left|T_{2}\right|\right\|=\left\|T_{2}^{2}\right\|=\left\|T^{2}\right\|_{e} .
$$

Similarly $\|S\|=\|T\|_{e}$, so the proof is complete.

REMARK 8. One can see from this proof, that this approach does not extend to higher powers of $T$. The difficulty in simultaneously getting identical perturbations of two inside factors of $T^{4}$, in order to reduce the norm of $T^{4}$, seems to be beyond these techniques.

We have been unable to get the result in Theorem 7 only for the case where $T^{3}$ is compact and $T^{2}$ is not compact. The complication lies in finding a compact perturbation $S$ with both $S^{3}=0$ and $\|S\|=\|T\|_{e}$. On the one hand, this is a fairly special case, reducing to a $3 \times 3$ upper triangular operator matrix. On the other hand, it points up a general limitation involved in trying to combine the totally unrelated methods for perturbing compact and noncompact products. Our results are summarized in the following:

THEOREM 9. Let $T$ be any operator in $\mathscr{B}(\mathscr{H})$. Then $\left\|T^{2}\right\|_{e}$

(i) there is a compact perturbation $S$ with $\|S\|=\|T\|_{e}$ and $\left\|S^{2}\right\|=$ 
(ii) there is a compact perturbation $R$ with $\left\|R^{2}\right\|=\left\|T^{2}\right\|_{e}$ and $\left\|R^{3}\right\|=\left\|T^{3}\right\|_{e}$

(iii) if $T^{2}$ is compact or $T^{3}$ is not compact we can choose $S=R$.

Proof of (i). If $T^{2}$ is not compact we can argue as in the beginning of the previous proof. If $T^{2}$ is compact, then using Theorem 2.4 of [6], we get a compact perturbation $T_{1}$ of $T$ with $T_{1}^{2}=0$. Then $T_{1}$ is equivalent to an operator matrix $T_{1}=\left(\begin{array}{cc}0 & A \\ 0 & 0\end{array}\right)$, on a Hilbert space $\mathscr{E} \oplus \mathscr{E}$, with $\left\|T_{1}\right\|=\|A\|$. Let $A^{\prime}$ be a compact perturbation of $A$ with $\left\|A^{\prime}\right\|=$ $\|A\|_{e}$, then $S=\left(\begin{array}{cc}0 & A^{\prime} \\ 0 & 0\end{array}\right)$ satisfies (i).

Proof of (ii). If $T^{2}$ is compact, (i) applies. If $T^{3}$ is not compact, use the preceding theorem. Otherwise, let $T_{1}$ be a compact perturbation of $T$ with $T_{1}^{3}=0$ [6], so $T_{1}$ is equivalent to

$$
T_{1}=\left(\begin{array}{ccc}
0 & A & B \\
0 & 0 & C \\
0 & 0 & 0
\end{array}\right), \quad \text { with } \quad T_{1}^{2}=\left(\begin{array}{ccc}
0 & 0 & A C \\
0 & 0 & 0 \\
0 & 0 & 0
\end{array}\right)
$$

where $\left\|T_{1}^{2}\right\|=\|A C\| . \quad$ Apply Theorem 3 to $A C$ to get $\left\|A^{\prime} C\right\|=\|A C\|_{e}$, and set

$$
S=\left(\begin{array}{ccc}
0 & A^{\prime} & B \\
0 & 0 & C \\
0 & 0 & 0
\end{array}\right)
$$

Proof of (iii). By (i) and the previous theorem.

We remark that the full strength of Theorem 3 (and hence of Theorem 1) is not required for part (i) of Theorem 9. In particular, Theorem 1 can be proved much more easily if the factor $A=I$; and (i) follows readily from this case.

An operator $T \in \mathscr{B}(\mathscr{H})$ is a weighted shift of multiplicity $k$ if there is an orthonormal basis $\left\{e_{n}\right\}_{n}$ for $\mathscr{H}$ on which $T$ is defined by $T e_{n}=a_{n} e_{n+k}$, $n=1,2, \cdots$, where $\left\{a_{n}\right\}_{n}$ is a sequence of complex numbers. In order to prove our theorem for weighted shifts we need the following elementary lemma.

Lemma 10. Assume $\alpha>\beta>0$, and let $a_{1}, \cdots, a_{n}$ be an $n$-tuple of positive numbers with $\Pi a_{i}=\alpha$. Then there is an $n$-tuple of positive numbers $b_{1}, \cdots, b_{n}$ with $\Pi b_{i}=\beta ; b_{i} \leqq a_{i}$ all $i ;$ and $\max \left(a_{i}-b_{i}\right) \leqq$ $\alpha^{1 / n}-\beta^{1 / n}$. 
Proof. If there is some $i$ with $a_{i}<\alpha^{1 / n}-\beta^{1 / n}$ the result is trivial. So assume $a_{i} \geqq \alpha^{1 / n}-\beta^{1 / n}$, all $i$; then $a_{i} \leqq \alpha /\left(\alpha^{1 / n}-\beta^{1 / n}\right)^{n-1}$.

Now, define $\left(c_{1}, \cdots, c_{n}\right)$ by $c_{i}=a_{i}-\left(\alpha^{1 / n}-\beta^{1 / n}\right)$. To finish the proof, it suffices to show that $\Pi c_{i} \leqq \beta$. For, by the continuity of the product, we can then find $\left(b_{1}, \cdots, b_{n}\right)$ with $c_{i} \leqq b_{i} \leqq a_{i}$ all $i$, and $\Pi b_{i}=\beta$.

Set $\gamma=\alpha^{1 / n}-\beta^{1 / n}$ and consider the function $f\left(a_{1}, \cdots, a_{n}\right)=\Pi c_{i}=$ $\Pi\left(a_{i}-\gamma\right)$ defined on the compact set $X$ of $\mathbf{R}^{n}$ where $\gamma \leqq a_{i} \leqq \alpha / \gamma^{n-1}$ and where $\Pi a_{1}=\alpha$. Then $f$ has a maximum value $M$ on $X$ : suppose it occurs at $\left(a_{1}, \cdots, a_{n}\right)$ with $a_{1}>a_{2}$. Consider $\left(\sqrt{a_{1} a_{2}}, \sqrt{a_{1} a_{2}}\right.$, $\left.a_{3}, \cdots, a_{n}\right) \in X$. Note that $\sqrt{a_{1} a_{2}}<\frac{1}{2}\left(a_{1}+a_{2}\right)$. Thus

$$
\begin{aligned}
f\left(\sqrt{a_{1} a_{2}}, \sqrt{a_{1} a_{2}}, a_{3}, \cdots, a_{n}\right) & =\left(\sqrt{a_{1} a_{2}}-\gamma\right)^{2} \prod_{3}^{n}\left(a_{i}-\gamma\right) \\
& >\left(a_{1} a_{2}-\gamma\left(a_{1}+a_{2}\right)+\gamma^{2}\right) \prod_{3}^{n}\left(a_{i}-\gamma\right) \\
& =\Pi\left(a_{i}-\gamma\right)=M,
\end{aligned}
$$

a contradiction. Hence $a_{1}=a_{2}$, and by symmetry, $f$ takes its maximum at $\left(\alpha^{1 / n}, \cdots, \alpha^{1 / n}\right)$, so $M=\beta$. The lemma is proved.

THEOREM 11. Let $T$ be a weighted shift. Then there is a compact perturbation $S$ of $T$ with $\left\|S^{n}\right\|=\left\|T^{n}\right\|_{e}$, for all $n=1,2, \cdots$.

Proof. Let $T$ be a shift with weight sequence $\left(a_{j}\right)_{j}$. We give the proof for a shift of multiplicity 1: in this case,

$$
\left\|T^{n}\right\|=\sup _{J}\left|a_{j} a_{j+1} \cdots a_{J+n-1}\right|
$$

The proof for $T$ of multiplicity $k$ is similar, where

$$
\left\|T^{n}\right\|=\sup _{j}\left|a_{j} a_{j+k} \cdots a_{j+(n-1) k}\right|
$$

A straightforward computation allows us to assume $\|T\| \leqq 1$. Let $\mu_{n}=\left\|T^{n}\right\|, \quad \nu_{n}=\left\|T^{n}\right\|_{e}, n=1,2, \cdots$. We will define by induction a sequence $\left\{S_{n}\right\}_{n}$ of weighted shifts, each obtained by reducing the moduli of the weights of the preceding, and which converges to the desired perturbation $S$ of $T$.

Let $S_{1}$ be the shift with weights $\left\{a_{1 j}\right\}_{\text {; }}$, where 


$$
a_{1 j}=\left\{\begin{array}{lll}
a_{j} & \text { if } & \left|a_{j}\right| \leqq \nu_{1} \\
a_{j} \frac{\nu_{1}}{\left|a_{j}\right|} & \text { if } & \left|a_{j}\right|>\nu_{1} .
\end{array}\right.
$$

Then $\left\|S_{1}\right\|=\nu_{1}=\|T\|_{e}$, and $\left|a_{1 j}-a_{j}\right|$ is a sequence converging to zero and hence $T-S_{1}$ is compact.

Assume for induction, that $S_{1}, S_{2}, \cdots, S_{n-1}$ have been constructed so that for each $k=1,2, \cdots, n-1$, and for each $j \leqq k$ :

(i) $T-S_{k}$ is compact;

(ii) $\left\|S_{k}^{k}\right\|=\nu_{k}$;

(iii) $\left\|S_{j}-S_{k}\right\| \leqq \max \left\{\mu_{j}^{1 / j}-\nu_{j}^{1 / j} \cdots \mu_{k}^{1 / k}-\nu_{k}^{1 / k}\right\}$;

(iv) if $S_{j}$ and $S_{k}$ have weights $\left\{a_{j i}\right\}_{i}$ and $\left\{a_{k i}\right\}_{i}$ resp., then $\left|a_{j i}\right| \geqq\left|a_{k i}\right|$, each $i$.

Construct $S_{n}$ as follows: note that $\left\|T^{n}\right\|=\sup _{j}\left|a_{j} a_{j+1} \cdots a_{j+n-1}\right|=\mu_{n}$. Let $\Lambda$ be the set of $j$ with $\left|a_{j} \cdots a_{j+n-1}\right|>\nu_{n}$. Define $\gamma_{j}=\left|a_{j} \cdots a_{j+n-1}\right|$, for $j \in \Lambda$. Then

$$
\lim _{i} \gamma_{j}=\left\|T^{n}\right\|_{e}=\nu_{n} .
$$

Applying the preceding Lemma, we see that for each $j \in \Lambda$, there is an $n$-tuple $\left(b_{j}, b_{j+1}^{(1)}, b_{j+2}^{(2)}, \cdots, b_{j+n-1}^{(n-1)}\right)$ satisfying:

(1) $\left|b_{j} b_{j+1}^{(1)} \cdots b_{j+n-1}^{(n-1)}\right|=\nu_{n}$;

(2) $\left|b_{j}\right| \leqq\left|a_{j}\right|,\left|b_{j+i}^{(i)}\right| \leqq\left|a_{j+i}\right|, i=1,2, \cdots, n-1$;

(3) $\max \left\{\left|a_{j}-b_{j}\right|, \cdots,\left|a_{j+i}-b_{j+1}^{(i)}\right|\right\} \leqq \gamma_{j}^{1 / n}-\nu_{n}^{1 / n}$.

Choose $c_{j}$ to be one among $a_{j}$ and those of $b_{j}, b_{j}^{(1)}, \cdots, b_{j}^{(n-1)}$ which are defined, having a minimum modulus (note that since $\gamma_{j}$ is only defined for $j \in \Lambda$, some of the $b_{j}, b_{j}^{(i)}$ may not be defined). Let $T_{n}$ be the shift with weights $\left\{c_{j}\right\}_{j}$.

Note that $T-T_{n}$ is compact, since either $a_{j}=c_{\jmath}$, or

$$
\left|a_{j}-c_{j}\right| \leqq \max _{k}\left\{\gamma_{k}^{1 / n}-\nu_{n}^{1 / n}: k \in \Lambda \quad \text { with } \quad k=j-n+1, \cdots, j\right\},
$$

where $\lim _{j} \gamma_{j}=\nu_{n}$. This inequality also shows that $\left\|T-T_{n}\right\| \leqq \mu_{n}^{1 / n}-\nu_{n}^{1 / n}$, since $\mu_{n}=\sup \gamma_{k}$. Also, $\left\|T_{n}^{n}\right\|=\nu_{n}$.

Define $S_{n}$ to be the shift with weights $\left\{a_{n j}\right\}_{j}$ where $a_{n j}$ is the one of $a_{n-1, j}$ and $c_{j}$ having minimum modulus.

Clearly $T-S_{n}$ is compact; $\left|a_{n i}\right| \leqq\left|a_{n-1, i}\right|$ all $i=1,2, \cdots ;$ and $\left\|S_{n}^{n}\right\|=$ $\nu_{n}$. Also, we see that

$$
\left\|S_{j}-S_{n}\right\| \leqq \max \left\{\mu_{j}^{1 / j}-\nu_{j}^{1 / j}, \cdots, \mu_{n}^{1 / n}-\nu_{n}^{1 / n}\right\},
$$

by comparing the $i$ th weights of these operators: since $\left\|T-S_{n}\right\| \leqq$ $\mu_{n}^{1 / n}-\nu_{n}^{1 / n}$, since $\left|a_{n 1}\right| \leqq\left|a_{n-1, i}\right|$ all $i$, and by induction hypothesis (iii). 
So, the sequence $\left\{S_{n}\right\}_{n}$ is constructed, and we will now see that it converges uniformly to some bounded operator $S$. The spectrum of any shift is circularly symmetric about the origin [5, p. 43]. Thus $\partial \sigma(T)$ consists of one or more circles. Now $\sigma(T)$ contains the spectrum of $\pi(T)$ in the Calkin algebra. By a theorem of C. Putnam [9], $\partial \sigma(T) \subset$ $\sigma(\pi(T)) \cup\{$ isolated eigenvalues of $T$ of finite multiplicity\}. Thus we conclude that $\sigma(T)$ and $\sigma(\pi(T))$ have the same radius. Thus

$$
\lim \left\|T^{n}\right\|^{1 / n}=\lim \left\|\pi(T)^{n}\right\|^{1 / n}=\lim \left\|T^{n}\right\|_{e}^{1 / n},
$$

so $\lim \mu_{n}^{1 / n}-\nu_{n}^{1 / n}=0$. Hence property (iii) implies that $\left\{S_{n}\right\}_{n}$ is uniformly Cauchy; so set $\lim S_{n}=S$. Then $T-S_{n}$ converges to a compact operator, $T-S$. From the construction of $\left\{S_{n}\right\}_{n}$, in particular property (iv), it is clear that $S$ is a shift whose $j$ th weight has modulus $\leqq$ the modulus of the $j$ th weight of each $S_{n}$. Thus, $\left\|S^{n}\right\| \leqq\left\|S_{n}^{n}\right\|=\nu_{n}$, each $n=1,2, \cdots$, and the result is proved.

The best possible result is attainable for operators which are direct sums of matrices of bounded degree.

THEOREM 12. Let $T=\sum_{k=1}^{\infty} \oplus T_{k}, a$ direct sum of $m \times m$ matrices. Then there is a compact perturbation $S$ of $T$ such that $\|p(S)\|=$ $\|p(T)\|_{e}$, for every complex polynomial $p$.

Proof. Consider each $T_{k}$ as an element of $\mathbf{C}^{m^{2}}$. Since $T$ is a bounded operator, the set $\left\{T_{k}\right\}_{k}$ is a bounded set in $\mathbf{C}^{m^{2}}$, so that the set $X \subset \mathbf{C}^{m^{2}}$ of accumulation points of $\left\{T_{k}\right\}_{k}$ is a compact set. We include in $X$ any $T_{k}$ which are repeated infinitely many times. Then $\left\{T_{k}\right\} \mid X$ has no accumulation points, so that if

$$
d_{k}=\operatorname{distance}\left(T_{k}, X\right)
$$

then $\lim d_{k}=0$. For each $T_{k}$ choose some $S_{k} \in X$ with $\left\|T_{k}-S_{k}\right\|=d_{k}$ (since all topologies on $\mathbf{C}^{n^{2}}$ are equivalent, we simply use the operator norm).

Let $S=\sum_{k=1}^{\infty} \oplus S_{k}$. Clearly $S$ is a compact perturbation of $T$. Furthermore, every element of the set $\left\{S_{k}\right\}_{k} \subset \mathbf{C}^{n^{2}}$ is an accumulation point of that set or occurs infinitely often, and thus for any complex polynomial $p$, the same is true for the set $\left\{p\left(S_{k}\right)\right\}_{k}$. Therefore

$$
\begin{aligned}
\|p(S)\| & =\left\|\sum_{k} \oplus p\left(S_{k}\right)\right\|=\sup _{k}\left\|p\left(S_{k}\right)\right\| \\
& =\lim \sup _{k}\left\|p\left(S_{k}\right)\right\|=\lim \sup _{k}\left\|p\left(T_{k}\right)\right\| .
\end{aligned}
$$


Let $E_{n}=\sum_{k=1}^{n} \oplus I_{k}$. Now, any compact operator $K$ satisfies

$$
\lim _{n}\left\|\left(I-E_{n}\right) K\left(I-E_{n}\right)\right\|=0
$$

Thus

$$
\begin{aligned}
\|p(T)+K\| & \geqq \lim \sup _{n}\left\|\left(I-E_{n}\right)(p(T)+K)\left(I-E_{n}\right)\right\| \\
& =\lim \sup _{n}\left\|\left(I-E_{n}\right) p(T)\left(I-E_{n}\right)\right\| \\
& =\lim \sup _{k}\left\|p\left(T_{k}\right)\right\| \\
& =\|p(S)\|,
\end{aligned}
$$

so $\|p(S)\|=\|p(T)\|_{e}$

COROLlARY 13. If $T \in \mathscr{B}(\mathscr{H})$ is a nilpotent weighted shift, there is a

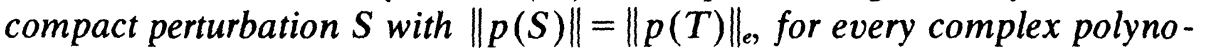
mial $p$.

Proof. Any such $T$ satisfies the hypotheses of Theorem 12.

COROLlary 14. Let $T$ be an n-normal operator. Then there is a compact perturbation $S$ such that $\|p(S)\|=\|p(T)\|_{e}$ for every complex polynomial $p$.

Proof. The operator $T$ may be regarded as an $n \times n$ operator matrix whose entries are commuting normal operators $\left\{T_{j}\right\}$ on a Hilbert space $\mathscr{E}$. It follows by a theorem of L. G. Brown, R. G. Douglas, and P. A. Fillmore [2, Corollary 5.4 , p. 83] that there is an orthonormal basis of $\mathscr{E}$ with each $T_{j}=D_{j}+K_{j}$, where $D_{j}$ is diagonal relative to this basis, for every $j=1,2, \cdots, n^{2}$, and where $K_{j}$ is compact. Let $K$ be the $n \times n$ operator matrix whose entries are the $K_{j}, j=1, \cdots, n^{2}$. Then $S=T-K$ is an $n \times n$ operator matrix with simultaneously diagonal entries $D_{j}$, so that $S$ is unitarily equivalent to an infinite direct sum of $n \times n$ matrices, and the previous theorem applies.

\section{REFERENCES}

1. S. K. Berberian, The Weyl spectrum of an operator, Indiana Univ. Math. J., 20 (1970/71), 529-544.

2. L. G. Brown, R. G. Douglas, and P. A. Fillmore, Unitary equivalence modulo the compact operators and extensions of $C^{*}$-algebras, Proceedings of a Conference on Operator Theory, Lecture Notes in Mathematics, Vol. 345, Springer-Verlag, New York, 1973; 58-128. 
3. S. R. Caradus, Query No. 65, Notices Amer. Math. Soc., 22 (1975), 198.

4. I. C. Gohberg and M. G. Krein, Introduction to the theory of linear nonselfadjoint operators, Translations of Mathematical Monographs, 18, Amer. Math. Soc., Providence, R. I., 1969.

5. P. R. Halmos, A Hilbert Space Problem Book, Van Nostrand, Princeton, N. J., 1967.

6. C. L. Olsen, A structure theorem for polynomially compact operators, Amer. J. Math., 93 (1971), 686-698.

7. C. L. Olsen and J. K. Plastiras, Quasialgebraic operators, compact perturbations, and the essential norm, Michigan Math. J., 21 (1974), 385-397.

8. G. K. Pedersen, Spectral formulas in quotient $C^{*}$-algebras, Copenhagen University, Preprint Series 1975, No. 22.

9. C. R. Putnam, The spectra of operators having resolvants of first-order growth, Trans. Amer. Math. Soc., 133 (1968), 505-510.

10. H. Weyl, Über beschrankte quadratischen Formen deren Differenz vollstetig ist, Rend. Circ. Mat. Palermo, 27 (1909), 373-392.

11. T. T. West, The decomposition of Riesz operators, Proc. London Math. Soc., (3) 16 (1966), $737-752$.

Received August 4, 1975. This research was supported in part by National Science Foundation Grant No. PO37621.

STATE UNIVERSITY-BUfFalo

AMHERST, NY 14226 



\section{Pacific Journal of Mathematics}

Vol. 68, No. 1

March, 1977

Richard Julian Bagby, On $L^{p}, L^{q}$ multipliers of Fourier transforms . .......

Robert Beauwens and Jean-Jacques Van Binnebeek, Convergence theorems in

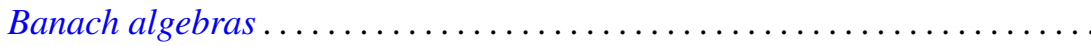

James Cyril Becker, Skew linear vector fields on spheres in the stable

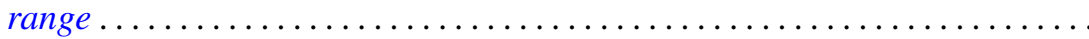

Michael James Beeson, Continuity and comprehension in intuitionistic formal

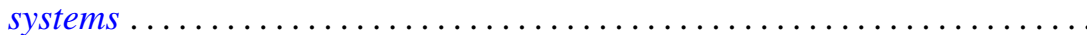

James K. Deveney, Generalized primitive elements for transcendental field

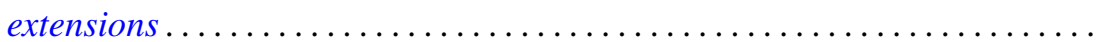

Samuel S. Feder, Samuel Carlos Gitler and K. Y. Lam, Composition properties

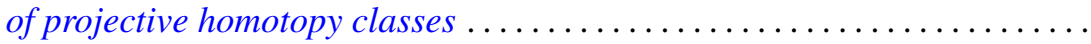

Nathan Jacob Fine, Tensor products of function rings under composition ......

Benno Fuchssteiner, Iterations and fixpoints . . . . . . . . . . . . . .

Wolfgang H. Heil, On punctured balls in manifolds

Shigeru Itoh, A random fixed point theorem for a multivalued contraction

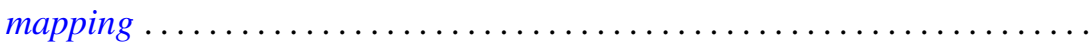

Nicolas P. Jewell, Continuity of module and higher derivations . . . . . . ......

Roger Dale Konyndyk, Residually central wreath products . . . . . . . . . . .

Linda M. Lesniak and John A. Roberts, On Ramsey theory and graphical

parameters.

Vo Thanh Liem, Some cellular subsets of the spheres.

Dieter Lutz, A perturbation theorem for spectral operators

P. H. Maserick, Moments of measures on convex bodies ... . . .

Stephen Joseph McAdam, Unmixed 2-dimensional local domains . .

D. B. McAlister and Norman R. Reilly, E-unitary covers for inverse semigroups...

William H. Meeks, III and Julie Patrusky, Representing codimension-one

homology classes by embedded submanifolds . . .

Premalata Mohapatro, Generalised quasi-Nörlund summability . .

Takahiko Nakazi, Superalgebras of weak-*Dirichlet algebras .

Catherine Louise Olsen, Norms of compact perturbations of operators .

William Henry Ruckle, Absolutely divergent series and isomorphism of

subspaces. II.

Bernard Russo, On the Hausdorff-Young theorem for integral operators .

Arthur Argyle Sagle and J. R. Schumi, Anti-commutative algebras and

homogeneous spaces with multiplications ............

Robert Evert Stong, Stiefel-Whitney classes of manifolds .

D. Suryanarayana, On a theorem of Apostol concerning Möbius functions of

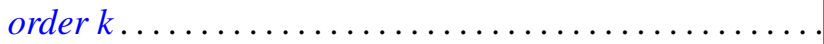

Yoshio Tanaka, On closedness of $C$ - and $C^{*}$-embeddings . . 